\title{
12 ANALYSIS OF ACPA POSITIVITY AND ACPA FINE SPECIFICITIES IN A LARGE SWEDISH TWIN COHORT (TWINGENE)
}

Aase HajHensvold, ${ }^{1}$ PatrikKE Magnusson, ${ }^{2}$ Monika Hansson, ${ }^{1}$ Lena Israelsson, ${ }^{1}$ Cecilia Carlens, ${ }^{1}$ Rikard Holmdahl, ${ }^{3}$ Per-Johan Jakobsson, ${ }^{1}$ Johan Askling, ${ }^{4}$ Tomas Ekström, Vivianne Malmström, ${ }^{1}$ Lars Klareskog, ${ }^{1}$ Anca Irinel Catrina' ${ }^{1}$ Rheumatology unit, Karolinska Institutet, Karolinska University Hospital, Stockholm, Sweden; ${ }^{2}$ Swedish Twin Registry Karolinska Institutet, Stockholm, Sweden; ${ }^{3}$ Medical Biochemistry and Biophysics Karolinska Institutet, Stockholm, Sweden; ${ }^{4}$ Clinical Epidemiology Unit, Dept of Medicine, Karolinska Institutet, Stockholm, Sweden; ${ }^{5}$ Department of Clinical Neuroscience, Karolinska Institutet, Karolinska University Hospital, Stockholm, Sweden

10.1136/annrheumdis-2011-201231.12

Background and objectives Genetic factors play a significant role in the variance of susceptibility for anticitrullinated protein antibodies (ACPA)-positive RA (Woude et al 2009). The authors wanted to study ACPA positivity as an entity and aimed to investigate the influence of genetic and environmental factors for ACPA positivity using a twin study approach.

Methods The authors used a subset of the Swedish twin registry, including 12594 monozygotic (MZ) and dizygotic (DZ) twins born 1958 or earlier. Serum samples were analysed for ACPA using a commercial ELISA (cyclic citrullinated peptides 2). Positive samples were also run for corresponding cyclic arginine peptide. Next, the ACPA-positive samples were investigated for ACPA fine specificities against native and citrullinated forms of $\alpha$-enolase, collagen type II, vimentin and fibrinogen peptides. RA was verified by data from national care registers and/or by reviewing medical records. 6252 twins were defined as smokers according to information from questionnaire. Epigenetic changes such as DNA methylation, were measured in peripheral blood DNA from 20 monozygotic RA discordant twin pairs using the 'whole genome CHARM approach'.

Results 387 of 12594 tested individuals (3.1\%) were positive for ACPA ( $\geq 25 \mathrm{AU} / \mathrm{ml}) .202$ of the 387 displayed high titres $(3 \times$ above cut off). High reactivity to arginine (optical density $>0.4)$ were detected in 14 twins. Almost half $(45 \%)$ of the ACPA positive samples had reactivity to any of the tested citrullinated peptides. Reactivity against Cit-enolase and Citvimentin were most common, detected in 39\% respectively $23 \%$. Among the ACPA-positive, 313 twin complete pairs were available, 7 concordant (all same sexed) and 306 ACPA discordant pairs. Concordance for ACPA was $4 \%$ among MZ 
and $3 \%$ among DZ twin pairs, with no significant tetrachoric correlation (r MZ twins $0.17,95 \%$ CI 0.10 to 0.44 , and r DZ twins $0.14,95 \%$ CI 0.10 to 0.36 ). Concordance for high titre positivity was $5 \%$ among $\mathrm{MZ}$ and $3 \%$ among $\mathrm{DZ}$ twins. The concordance rate for Cit-enolase, based on the data from the ACPA positive subgroup, was $11 \%$ among $M Z$ and $5 \%$ among DZ twins. Smokers had an increased risk of developed ACPA as compared with non-smokers (OR 1.33, 95\% CI 1.08 to 1.63). Preliminary results from epigenetic study of monozygotic ACPA positive RA-positive twins compared to a healthy co twin identified 50 genes associated with differentially methylated regions (false discovery rate $<0,05$ ).

Conclusions Our results indicate that environment may be more important than genetics in determining which individuals will develop ACPA. 